\title{
A Comparison of Predictive Parameter Estimation using Kalman Filter and Analysis of Variance
}

\author{
Asim ur Rehman Khan, Haider Mehdi, Syed Muhammad Atif Saleem, Muhammad Junaid Rabbani \\ Multimedia Labs, \\ National University of Computer and Emerging Sciences (NUCES), \\ Pakistan
}

\begin{abstract}
The design of a controller significantly improves if internal states of a dynamic control system are predicted. This paper compares the prediction of system states using Kalman filter and a novel approach analysis of variance (ANOVA). Kalman filter has been successfully applied in several applications. A significant advantage of Kalman filter is its ability to use system output to predict the future states. It has been observed that Kalman filter based predictive controller design outperforms many other approaches. An important drawback of such controllers is however that their performances deteriorate in situations where the system states have no correlation with the output. This paper takes a hypothetical model of a helicopter and builds system model using the statespace diagram. The design is implemented using SIMULINK. It has been observed that in situations where the states are dependent on system output, the ANOVA based state prediction gives comparable results with that of Kalman filter based parameter estimation. The ANOVA based parameter prediction, however outperforms Kalman filter based parameter prediction in situations where the output does not directly contribute in a particular state. The research was based on empirical results. Rigorous testing was performed on four internal states to prove that ANOVA based predictive parameter estimation technique outperforms Kalman based parameter estimation in situations where the system internal states is not directly linked with the output.
\end{abstract}

Keywords-Analysis of variance (ANOVA); Kalman controllers; predictive controller

\section{INTRODUCTION}

A linear time-invariant (LTI) continuous system can be inherently stable if all of its poles are on the left-hand side of s-plane. If, however some of the poles are on the right-hand side then it needs a controller to ensure that the poles in the right-hand side are cancelled by the zeros of the controller, making an inherently unstable system to become stable. Alternately a system may be inherently stable, but at higher gains its poles may move towards the right-hand side of splane. In case of a discrete system, the condition of stability requires the presence of poles within a unit circle resulting in a stable system. This research compares the estimates of Kalman filter based predictive parameter estimates with that of the ANOVA based predictive parameter estimates. The controllers are generally categorized as feedback controllers, adaptive controllers, and predictive controllers. Among these controllers, the predictive controller influences the activity of the system to adjust various parameters to achieve the targeted value. The system tracks output such that the difference between the desired and actual output remains within limits as per given matric. The predictive controller uses current output/states to adjust the parameters of system to change the future output/states. The controller is based on proactive approach. The time-series analysis predictive controller performs reasonably well in case of where the variations are relatively free from noise. One of known time series predictor is an auto-regressive (AR) controller. The prediction based on analysis of variance (ANOVA) is relatively new in predictive controller design. A significant advantage of using ANOVA is that the noise carried by the parametric variation is also accounted for in the model. A combination of auto-regressive model and ANOVA are successfully used to predict the computer utilization in an internet service provider (ISP) [1].

An adaptive controller design based on Kalman filter has provided an optimum control design during the last many decades. The implementation of Kalman filter observer for multivariable ship control system is discussed in [2]. In addition, the application of extended Kalman filter observer to estimate the state of time varying disturbance for robotic manipulator and industrial heating system is presented in [3][4]. An adaptive Kalman filter for state-of-charge (SOC) lithium-ion battery is discussed in [5]. Several other Techniques of predicting states using the Kalman filter have been discussed in [6]-[8].

This system uses more sophisticated state-space model to monitor the system states. If fast moving applications, the predictive controller performs better than the adaptive controller simply due to the fact that they predict future parameters based on past records. The proposed algorithm uses analysis of variance techniques (ANOVA) to predict the future states. The results further show that in certain situations the ANOVA based parameter prediction out performs that of Kalman filter based parameter estimation. Predictive controllers based on ANOVA can be used in real time control applications. To the best of our knowledge, no work has been done in this area. Future work may involve using other statistical techniques, like regression analysis, etc.

After a brief introduction in this section, the state-space model in continuous and discrete system of a hypothetical helicopter is discussed in Section 2. The Kalman filter based observer and parameter estimation is given in Section 3. The ANOVA based parameter estimation is given in Section 4. Section 5 gives several results that compare the estimated parameters using Kalman filter and the ANOVA based approaches. Section 6 concludes this paper. 


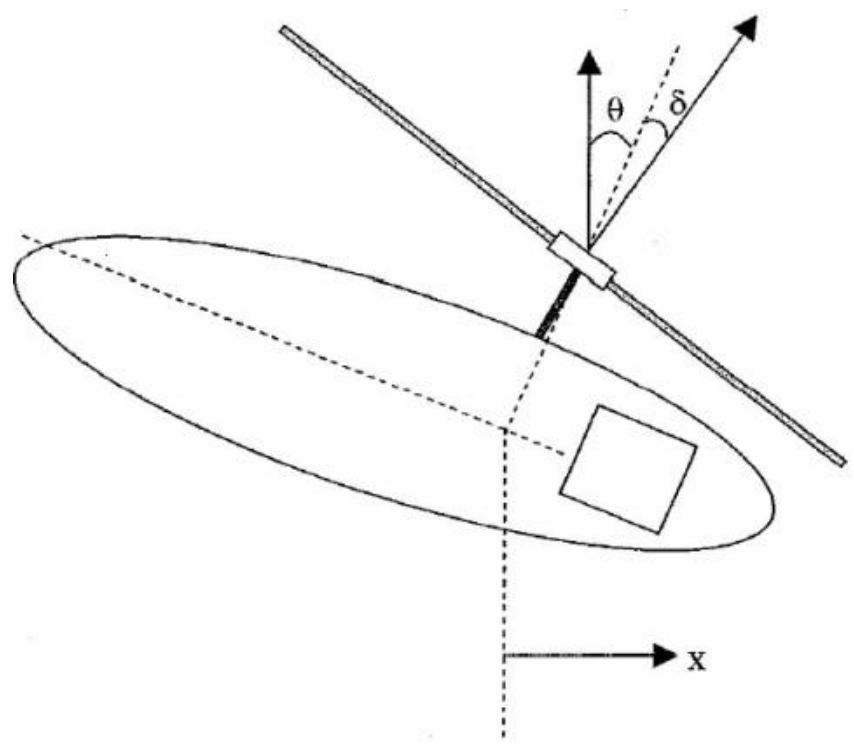

Fig. 1. A conceptual model of helicopter.

\section{MATHEMATICAL MODELS}

The model essentially consists of state-space model of a helicopter as in Fig. 1. The helicopter is expected to move only in the horizontal direction. The change in horizontal direction $x(t)$ is controlled by the input $\delta(t)$. The tilt in the horizontal direction is given by the angle $\theta(t)$. The model is described by using two second order equations.

$$
\begin{gathered}
\ddot{\theta}=-\tau_{1} \dot{\theta}-\alpha_{1} \dot{x}+\vartheta_{1} \delta \\
\ddot{x}=g \theta-\alpha_{2} \dot{\theta}-\tau_{2} \dot{x}+g \delta
\end{gathered}
$$

Where the first equation gives horizontal tilt, and the second equation gives helicopter position in the horizontal direction. All other parameters are coefficients with known and fixed values as given by

$$
\begin{array}{lcc}
\tau_{1}=0.415 & \alpha_{1}=0.0111 & \vartheta_{1}=6.27 \\
\tau_{2}=0.019 & \alpha_{2}=1.43 & g=9.81
\end{array}
$$

\section{A. Continuous Time State-Space Model}

The state-space model of a general system is represented by

$$
\begin{aligned}
& \dot{x}=A x+B u \\
& y=C x+D u
\end{aligned}
$$

Where, ' $x$ ' is the set of system states of a helicopter. The ' $\boldsymbol{u}$ ' is given input signal, and ' $\boldsymbol{y}$ ' is the output signal. The $\mathbf{A}, \mathbf{B}, \mathbf{C}$, and $\mathbf{D}$ are parameter of state-space model giving the system's characteristics. The bold letters represent matrices. The angle of tilt $\theta$ is represented by system state $x_{1}$. The derivative of tilt angle $(\dot{\theta})$ is equal to $x_{2}$. The horizontal position $x$ is represented by $x_{3}$, and the derivative of horizontal position $\dot{x}$ is represented by $x_{4}$.

$$
\left[\begin{array}{l}
x_{1} \\
x_{2} \\
x_{3} \\
x_{4}
\end{array}\right]=\left[\begin{array}{c}
\theta \\
\dot{\theta} \\
x \\
\dot{x}
\end{array}\right]
$$

The input $\mathrm{u}$ and the output state vector is defined as

$$
u=\delta, \text { and }\left[\begin{array}{l}
y_{1} \\
y_{2}
\end{array}\right]=\left[\begin{array}{l}
\theta \\
x
\end{array}\right]
$$

Where, $\delta$ is the amount of input signal, and the expected output $y_{1}$, and $y_{2}$ are the amount of horizontal motion and the amount of tilt. The state equations of a helicopter are given by the following set of equations:

$$
\begin{aligned}
& \dot{x}_{1}=\dot{\theta}=x_{2} \\
& \dot{x}_{2}=\ddot{\theta}=-0.415 x_{2}-0.0111 x_{4}+6.27 \delta \\
& \dot{x}_{3}=\dot{x}=x_{4} \\
& \dot{x}_{4}=\ddot{x}=9.81 x_{1}-1.43 x_{2}-0.0198 x_{4}+9.81 \delta
\end{aligned}
$$

These are represented in more compact form using matrices:

$$
\begin{gathered}
{\left[\begin{array}{l}
\dot{x}_{1} \\
\dot{x}_{2} \\
\dot{x}_{3} \\
\dot{x}_{4}
\end{array}\right]=\left[\begin{array}{cccc}
0 & 1 & 0 & 0 \\
0 & -0.415 & 0 & -0.0111 \\
0 & 0 & 0 & 1 \\
9.81 & -1.43 & 0 & -0.0198
\end{array}\right]\left[\begin{array}{l}
x_{1} \\
x_{2} \\
x_{3} \\
x_{4}
\end{array}\right]} \\
+\left[\begin{array}{c}
0 \\
6.27 \\
0 \\
9.81
\end{array}\right] \delta+v(k)
\end{gathered}
$$

The $v(k)$ represents undesired effects and this is approximately by a zero mean, normally distributed signal with constant variance. The output is given by

$$
\left[\begin{array}{l}
y_{1} \\
y_{2}
\end{array}\right]=\left[\begin{array}{llll}
1 & 0 & 0 & 0 \\
0 & 0 & 1 & 0
\end{array}\right]\left[\begin{array}{l}
x_{1} \\
x_{2} \\
x_{3} \\
x_{4}
\end{array}\right]+w(k)
$$

The D matrix in the standard state-space model is considered to be zero. The $w(k)$ is due to an undesired effect at the input, again approximated by a normally distributed signal having zero mean, and constant variance.

\section{B. State Space Model in Discrete Time}

The continuous state-state model is transformed into discrete state-space mode by using MATLAB routine. The sampling rate is $h=0.1$. A relatively smaller sampling rate results in large number of samples, and thus gives more accurate replica of the continuous state-space model. The general form of discrete state-space model is given by,

$$
\begin{aligned}
x(k+1) & =F x(k)+G u(k)+v(k) \\
y(k) & =C x(k)+w(k)
\end{aligned}
$$




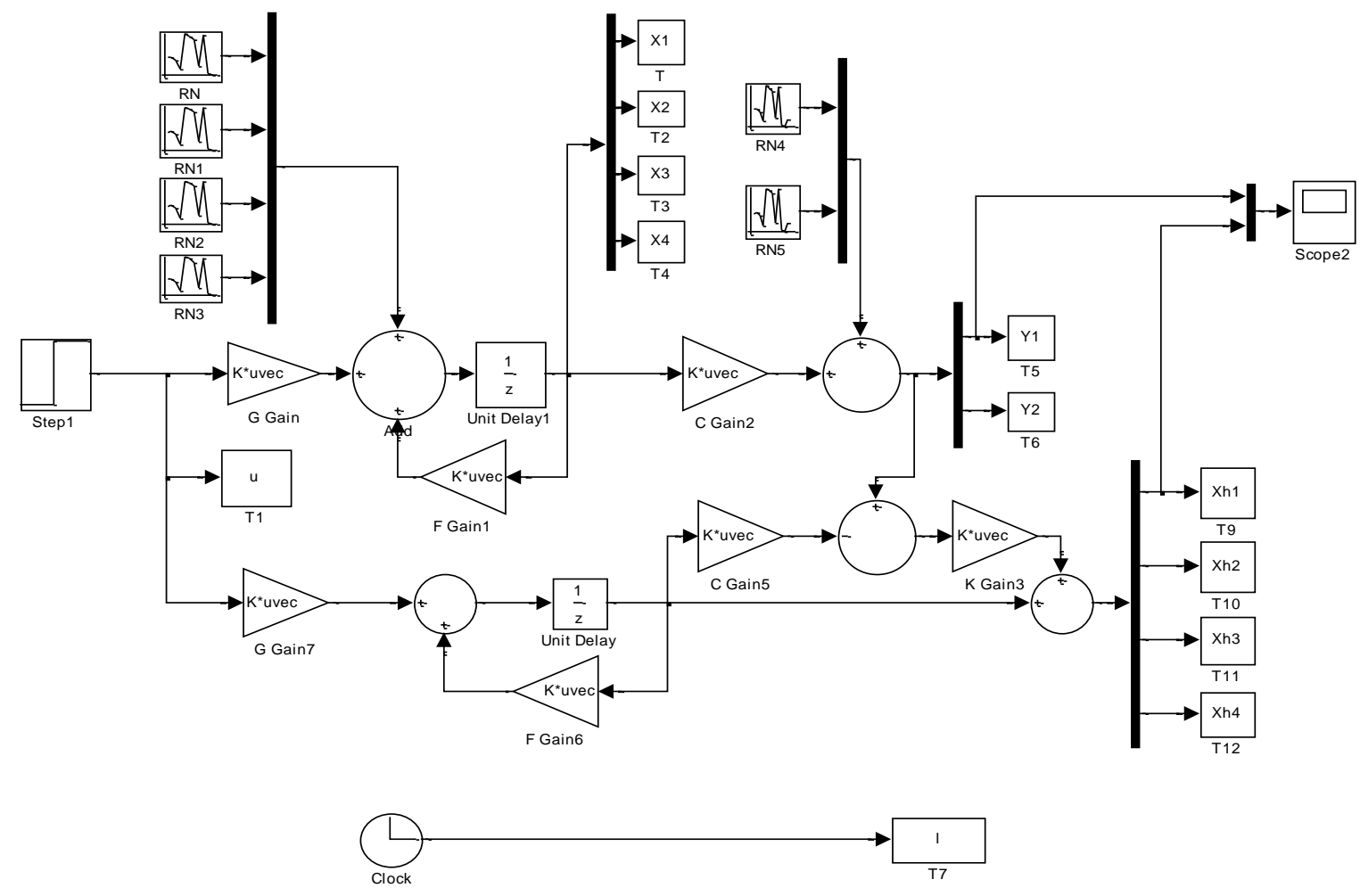

Fig. 2. SIMULINK model with Kalman filter observer.

The D matrix is again considered as zero. The $v(k)$ and $w(k)$ are corresponding process and measurement noises. The respective matrices are given by

$$
\begin{gathered}
F=\left[\begin{array}{cccc}
1.0000 & 0.0980 & 0 & -0.0001 \\
-0.0005 & 0.9594 & 0 & -0.0011 \\
0.0490 & -0.0054 & 1.0000 & 0.0999 \\
0.9801 & -0.0916 & 0 & 0.9981
\end{array}\right] \\
G=\left[\begin{array}{l}
0.0309 \\
0.6136 \\
0.0478 \\
0.9460
\end{array}\right] \quad C=\left[\begin{array}{llll}
1 & 0 & 0 & 0 \\
0 & 0 & 1 & 0
\end{array}\right]
\end{gathered}
$$

This model is given in Fig. 2 using SIMULINK.

\section{KALMAN FILTER OBSERVER}

The Kalman filter observer uses output signal to estimate various system states. A predictor based on these estimated state values can be designed to control the future states to minimize the amount of error. The estimated state has two components, a predicted state and a correction term based on the present output.

$$
\hat{x}(k)=\bar{x}(k)+K\{y(k)-C \bar{x}(k)\}
$$

Where, $\hat{x}(k)$ is the estimated value at sample $\mathrm{k}, \bar{x}(k)$ is the predicted state, and $K\{y(k)-C \bar{x}(k)\}$ is the correction term. $K$ is the gain matrix. The predicted state $\bar{x}(k)$ is

$$
\bar{x}(k)=F \hat{x}(k-1)+G u(k-1)
$$

The residual error is

$$
e(k+1)=x(k+1)-\hat{x}(k+1)
$$

The objective is to reduce the residual error. A system is controllable only if this is observable. The system is tested for observability and controllability using MATLAB routines. The positions of poles are also checked. It is found that some of the poles are outside the unit circle. The system is inherently unstable however with an appropriate controller design the system will become stable as all poles outside the unit circle are cancelled by zeros of controller. The Kalman gain matrix $\mathrm{K}$ is found using the MATLAB routine DLQE (discrete-linear-quadratic-estimator) command.

$$
[K, P]=D L Q E\left(F, H, C, R_{v}, R_{w}\right)
$$

Where, $K$ is the Kalman gain. The $\mathrm{H}$ is $3 \times 3$ identity matrix. $F$ and $C$ are previously defined. $R_{v}$ is the covariance matrix of system disturbance $\vartheta(k)$, and $R_{w}$ is the covariance matrix of output disturbance $w(k)$. These matrices are taken as,

$$
\begin{gathered}
R_{v}=\left[\begin{array}{cccc}
0.01 & 0 & 0 & 0 \\
0 & 0.01 & 0 & 0 \\
0 & 0 & 0.01 & 0 \\
0 & 0 & 0 & 0.01
\end{array}\right] \quad R_{w} \\
=\left[\begin{array}{cc}
0.01 & 0 \\
0 & 0.01
\end{array}\right]
\end{gathered}
$$


TABLE I. PERCENTAge ChANGES IN THE Four PARAMETERS USING KALMAN Filtering

\begin{tabular}{|c|c|c|c|c|c|c|c|c|c|c|c|c|c|}
\hline & & \multicolumn{3}{|c|}{$\mathrm{x} 1$} & \multicolumn{3}{|c|}{$\mathrm{x} 2$} & \multicolumn{3}{|c|}{$\mathrm{x3}$} & \multicolumn{3}{|c|}{$\mathrm{x4}$} \\
\hline S.No & Samples & Actual & x1_k & Error & Actual & x2_k & Error & Actual & x3_k & Error & Actual & x4_k & Error \\
\hline 1 & 25 & 11.8 & 9.4 & 2.4 & 5.6 & -9.5 & 15.0 & 149.4 & 104.7 & 44.7 & 166.2 & 148.8 & 17.3 \\
\hline 2 & 50 & 30.7 & 27.9 & 2.7 & 6.2 & -83.5 & 89.7 & 1114.8 & 887.8 & 227.1 & 672.6 & 980.4 & -307.8 \\
\hline 3 & 75 & 25.3 & 31.4 & -6.1 & -6.8 & -186.2 & 179.4 & 3672.7 & 3236.5 & 436.2 & 1362.7 & 2335.4 & -972.6 \\
\hline 4 & 100 & -5.6 & 0.7 & -6.3 & -19.7 & -217.6 & 197.9 & 7656.3 & 7188.3 & 468.0 & 1697.2 & 3044.5 & -1347.4 \\
\hline 5 & 125 & -61.1 & -58.3 & -2.7 & -19.1 & -123.4 & 104.2 & 11180.4 & 10950.9 & 229.5 & 893.1 & 1927.6 & -1034.6 \\
\hline 6 & 150 & -89.0 & -92.4 & 3.4 & 5.1 & 65.1 & -60.0 & 11157.9 & 11317.4 & -159.5 & -1032.8 & -1013.0 & -19.8 \\
\hline 7 & 175 & -13.9 & -28.1 & 14.2 & 47.7 & 178.0 & -130.3 & 6437.1 & 6732.2 & -295.1 & -2457.4 & -3442.1 & 984.7 \\
\hline 8 & 200 & 138.8 & 125.0 & 13.8 & 62.4 & -43.5 & 105.9 & 1249.0 & 933.2 & 315.8 & -1087.2 & -1491.5 & 404.4 \\
\hline 9 & 225 & 226.2 & 240.1 & -13.8 & 0.7 & -615.9 & 616.7 & 3886.0 & 2333.4 & 1552.5 & 3573.7 & 6154.7 & -2581.0 \\
\hline 10 & 250 & 94.6 & 140.4 & -45.8 & -103.2 & -1019.1 & 915.9 & 18898.0 & 16724.5 & 2173.5 & 7947.6 & 13948.9 & -6001.2 \\
\hline
\end{tabular}

TABLE II. Percentage Changes In the Four Parameters USING ANOVA

\begin{tabular}{|c|c|c|c|c|c|c|c|c|c|c|c|c|c|}
\hline & & \multicolumn{3}{|c|}{$\mathrm{x1}$} & \multicolumn{3}{|c|}{$\mathrm{x} 2$} & \multicolumn{3}{|c|}{$\mathrm{x} 3$} & \multicolumn{3}{|c|}{$\mathrm{x} 4$} \\
\hline S.No & Samples & Actual & x1_a & Error & Actual & x2_a & Error & Actual & x3_a & Error & Actual & x4_a & Error \\
\hline 1 & 25 & 11.8 & 9.8 & 2.0 & 5.6 & 3.5 & 2.0 & 149.4 & 134.2 & 15.2 & 166.2 & 159.0 & 7.1 \\
\hline 2 & 50 & 30.7 & 28.8 & 1.9 & 6.2 & 4.5 & 1.7 & 1114.8 & 1065.9 & 48.9 & 672.6 & 654.8 & 17.8 \\
\hline 3 & 75 & 25.3 & 25.5 & -0.2 & -6.8 & -6.8 & 0.0 & 3672.7 & 3590.6 & 82.1 & 1362.7 & 1352.6 & 10.1 \\
\hline 4 & 100 & -5.6 & -5.9 & 0.3 & -19.7 & -21.2 & 1.5 & 7656.3 & 7568.6 & 87.7 & 1697.2 & 1707.8 & -10.6 \\
\hline 5 & 125 & -61.1 & -61.3 & 0.3 & -19.1 & -20.6 & 1.4 & 11180.4 & 11160.9 & 19.5 & 893.1 & 931.3 & -38.3 \\
\hline 6 & 150 & -89.0 & -89.7 & 0.7 & 5.1 & 5.0 & 0.1 & 11157.9 & 11255.3 & -97.4 & -1032.8 & -991.4 & -41.4 \\
\hline 7 & 175 & -13.9 & -16.8 & 2.8 & 47.7 & 47.8 & -0.1 & 6437.1 & 6578.0 & -140.8 & -2457.4 & -2471.7 & 14.3 \\
\hline 8 & 200 & 138.8 & 135.0 & 3.7 & 62.4 & 62.9 & -0.5 & 1249.0 & 1248.7 & 0.4 & -1087.2 & -1186.4 & 99.2 \\
\hline 8 & 225 & 226.2 & 227.9 & -1.7 & 0.7 & 3.0 & -2.3 & 3886.0 & 3595.5 & 290.4 & 3573.7 & 3458.0 & 115.7 \\
\hline 10 & 250 & 94.6 & 104.1 & -9.5 & -103.2 & -99.4 & -3.7 & 18898.0 & 18432.6 & 465.4 & 7947.6 & 7945.2 & 2.4 \\
\hline
\end{tabular}

The Kalman gain $\mathrm{K}$ is found to be equal to

$$
K=\left[\begin{array}{cc}
0.6405 & 0.0006 \\
0.3695 & -0.1370 \\
0.0006 & 0.6683 \\
0.1669 & 0.8703
\end{array}\right]
$$

The state estimators using Kalman gain is found by

$$
\begin{gathered}
{\left[\begin{array}{l}
\hat{x}_{1}(k+1) \\
\hat{x}_{2}(k+1) \\
\hat{x}_{3}(k+1) \\
\hat{x}_{4}(k+1)
\end{array}\right]=\left[\begin{array}{l}
\bar{x}_{1}(k) \\
\bar{x}_{2}(k) \\
\bar{x}_{3}(k) \\
\bar{x}_{4}(k)
\end{array}\right]+G(k)} \\
G(k)=\left[\begin{array}{cc}
0.6405 & 0.0006 \\
0.3695 & -0.1370 \\
0.0006 & 0.6683 \\
0.1669 & 0.8703
\end{array}\right]\left\{\left[\begin{array}{l}
y_{1} \\
y_{2}
\end{array}\right]\right. \\
\left.-\left[\begin{array}{llll}
1 & 0 & 0 & 0 \\
0 & 0 & 1 & 0
\end{array}\right]\left[\begin{array}{l}
\bar{x}_{1}(k) \\
\bar{x}_{2}(k) \\
\bar{x}_{3}(k) \\
\bar{x}_{4}(k)
\end{array}\right]\right\}
\end{gathered}
$$

The system design including the Kalman filter observer is given in Fig 2.

\section{ANOVA PREDICTOR DESIGN}

The regression based predictive modeling has been used in several fields. The human behavior is predicted based on the known facts. The statistical based prediction has been used in marketing, financial services like banking \& insurance. It has also been used in the telecommunications industry. A software package, Statistical Analysis System (SAS) has been developed by SAS Institute that helps in the advance analysis like multivariate analyses, data management, business intelligence, and predictive analysis. The package also uses ANOVA to provide the necessary analysis. An ANOVA based prediction of moisture buildup in electronic enclosure is proposed in [9]. The prediction of dataset by software engineers using ANOVA is demonstrated in [10]. An Intrusion Detection System by feature elimination has been demonstrated in [11]. ANOVA based parameter prediction is also demonstrated in [12].

This paper is an enhanced version of a recently published conference paper [13]. The analysis of variance (ANOVA) based proposed model is given by,

$$
\hat{x}(k)=\hat{\mu}_{k}+\hat{\alpha}_{k}+\hat{\beta}_{k}+\epsilon(k)
$$

where $\hat{x}(k)$ is the estimated value of $\mathrm{k}^{\text {th }}$ sample. This is approximated by an estimated general mean $\hat{\mu}_{k}$, sample parameters $\hat{\alpha}_{k}$, and $\hat{\beta}_{k}$. The unknown effects are given in the last term $\epsilon(k)$. The sample mean and the other parameters are found using 


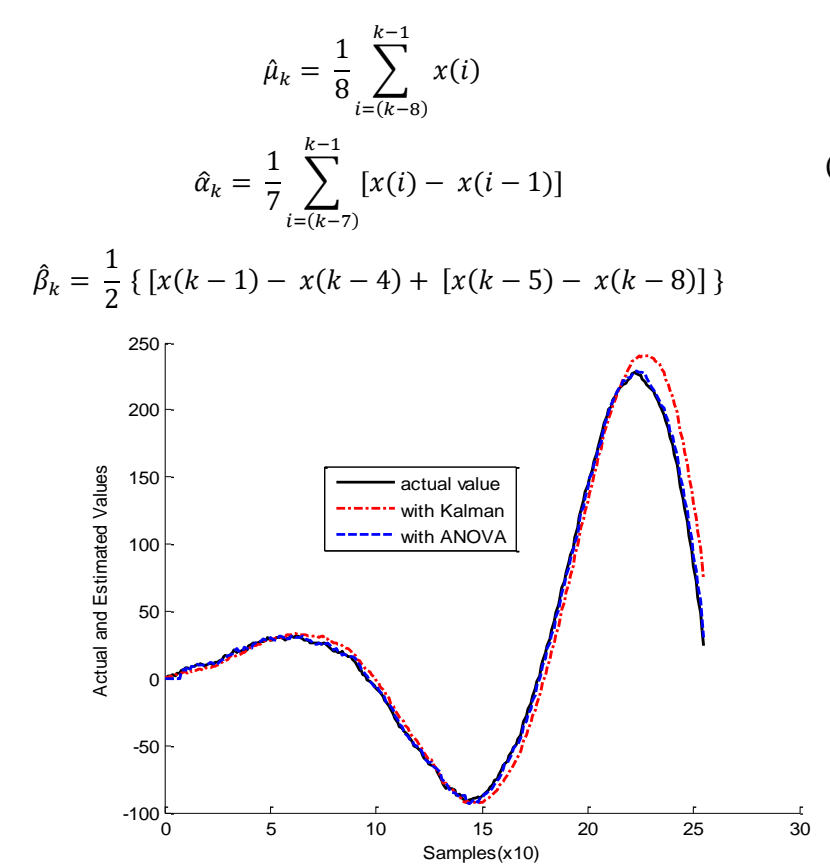

(a)

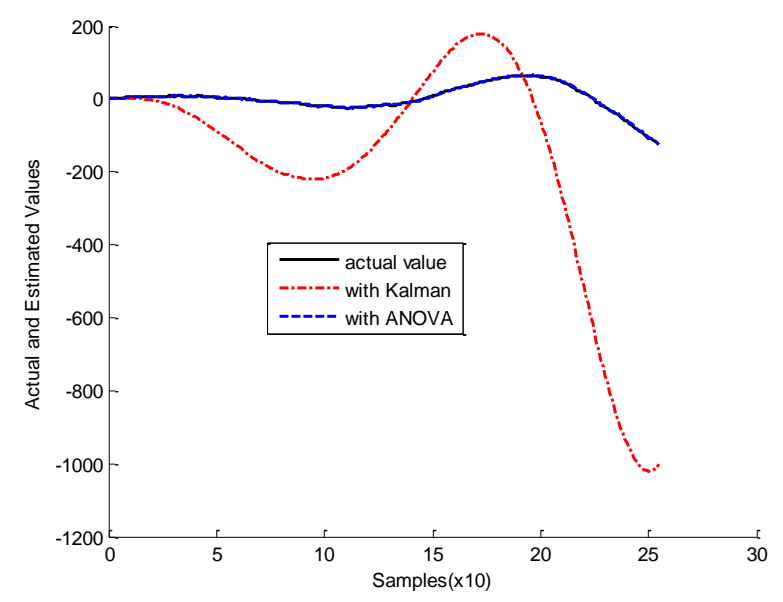

(b)

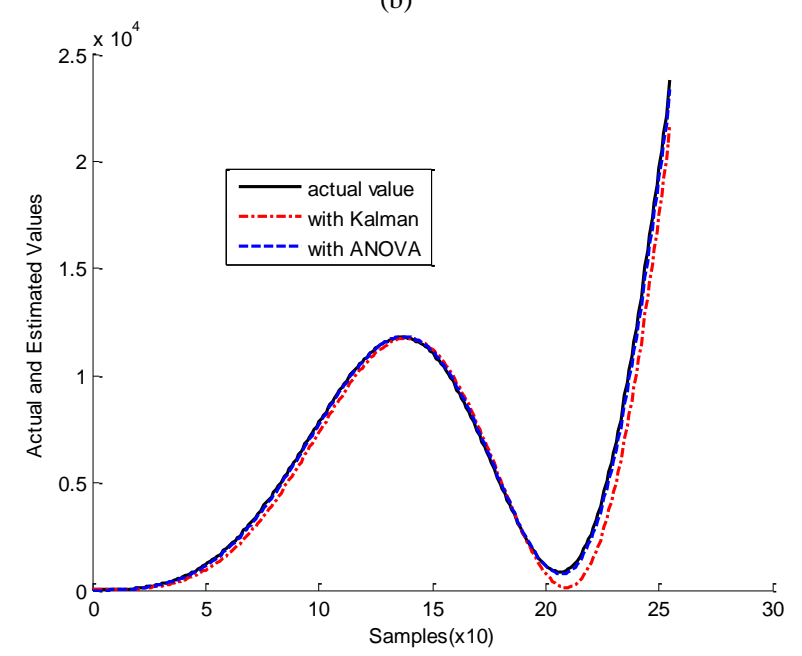

(c)

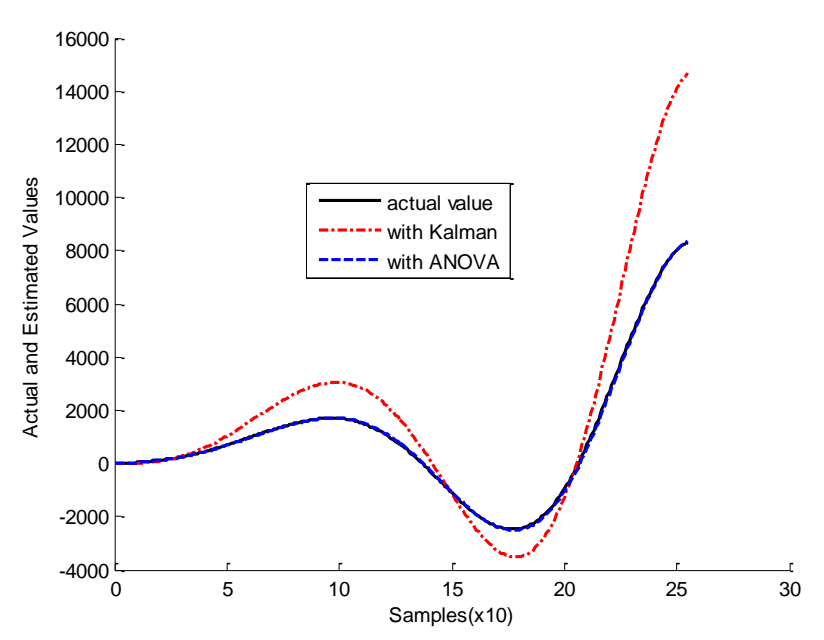

(d)

Fig. 3. (a)-(d) Actual and Estimated values of parameters, $x_{1}, x_{2}, x_{3}$, and $x_{4}$.

The above equation extracts three parameters based on sample mean of past eight samples $\mathrm{b} \hat{\mu}_{k}$, the mean difference of past eight samples by $\hat{\alpha}_{k}$, and the sample mean of past four samples with its preceding four samples.

\section{Simulation Results}

The main objective of this work is the prediction of four states that represent the motion of a helicopter. This is possible only due to state-space analysis. The states are initially in continuous domain which is converted into discrete model using the sampling rate of 0.1 . The sampling rate is critical in the sense that a smaller sample time results in more accurate analog-digital conversion, but results in much larger number of samples. Alternately a larger sampling rate results in coarse analog-digital conversion but a fewer samples. The most appropriate sampling rate depends on the application. It has been observed that a sampling rate of 0.1 results in most appropriate combination of refinement, and amount of data for a helicopter model. The output signal comprises of two parameters: an angle of tilt $(\theta)$ and the horizontal motion $(x)$ based on four internal states. These states are given as $x_{1}, x_{2}, x_{3}$, and $x_{4}$ as given in (4). The first step is the conversion of continuous state-space model into a discrete state-state model (9). The MATLAB routines are used to find matrices F, G, and C in discrete state-space model (10). These matrices, respectively, correspond to $\mathrm{A}, \mathrm{B}$, and $\mathrm{C}$ in continuous state-space model. Next it is desired to find if the system needs a controller, and if it is controllable. A discrete system is inherently stable if all of its poles are inside the unit circle. Such a system may only need a very simple controller that monitors system gain and ensures that the poles remain within the unit circle. If however, one or more poles are outside the unit circle then the system is inherently unstable, and sophisticated controller is required to cancel all poles that are outside the unit circle. It was found that the proposed model is not inherently stable, as it has one pole outside the unit circle. A second challenge is to learn if the system is indeed controllable. A system is controllable only if the internal states are observable. The validation of the observability is performed with a series of tests (14)-(17). The 
model is implemented by using SIMULINK. The original equations are based on a second order system. A significant advantage of using state-space analysis is that the model is implemented by using a single delay element, thus reducing the system order to one-dimension model. Other advantages of using discrete signal are the requirement of much smaller storage space, and the flexibility of using advanced signal processing techniques.

The original model and the Kalman filter estimate of states are given in Fig. 2. The actual values of selected samples, the estimated values using Kalman filter, and the error is given in Table 1. The graphically plots are given in Fig. 3(a)-(d). It is clear that the two states $x_{1}$, and $x_{3}$ are estimated with good accuracy. However, the other two states $x_{2}$, and $x_{4}$ are not estimated with good accuracy. The main reason is the fact that Kalman filter observer uses output signals $y_{1}$, and $y_{2}$ for the prediction of $x_{1}$, and $x_{3}$. It however, does not use output $y_{1}$ and $y_{2}$ for the prediction of other two states $x_{2}$, and $x_{4}$. This inherent shortcoming in Kalman based parameter estimator results in poor performance in $x_{2}$, and $x_{4}$.

The performance of ANOVA based estimator design is given in Fig. 3, where the estimators of states $x_{1}, x_{2}, x_{3}$, and $x_{4}$ are super imposed on the previous drawn estimates using Kalman filter. Each estimator is approximate by a time-series analysis. The analysis is based on a general mean, mean value of a first order gradient, and the mean value of gradient of a group of values as given in (18). The estimated values specific parameters are found using (19). The number of samples in this particular example is restricted to only 8 samples. It is expected that sampling time of 0.1 seconds in this example is small enough to highlight the minute change with sufficient number of samples. It is clear that any change in sampling time would change the matrices $F$, and $G$ which would change the Kalman gain matrix K, resulting in completely new model. The actual values of states, the estimated values using ANVOA, and the corresponding error of selected samples are given in Table 2. The graphical plots of these samples are given in Fig. 3(a)-(d). A comparison of Tables 1 and 2, and Fig. 3 clearly shows that both Kalman filter and ANOVA based parameter prediction performs reasonable well for states $x_{1}$, and $x_{3}$, however, the performance of ANOVA based parameter prediction is much superior to that of Kalman filter based parameter predictive estimation.

The list of parameters in an ANOVA design can be changed to suit a particular analysis. As an example, in this particular analysis the objective of this model was to have an estimator based on the mean value, and the gradients at two levels. The model was relatively simple as the estimator was based on the aggregation of three independent terms. This model can be further simplified by considering only the mean value, or made complicated by considering terms related to multiple effects.
A significant advantage of ANOVA based estimator is that these estimators are robust and are able to correctly estimation even if the states are corrupted with high degree of normally distributed noise.

\section{CONCLUSIONS}

This paper presents a model to estimate the internal states of a helicopter using analysis of variance (ANOVA). The results are compared with parameter estimation using Kalman filter. It has been observed that both the approaches yield comparable results when states have some form of dependencies on the system output. The ANOVA based approach however outperforms Kalman filter approach in situations where the internal states do not depend on the system output. The model is implemented on SIMULINK.

\section{REFERENCES}

[1] Wei Xu, Xiaoyun Zhu, Sharad Singhal, Zhikui Wang "Predictive control for dynamic resource allocation in entrepreneur data centers", IEEE/IFIP Network Operations and Management Symposium NOMS, pp. 115-126, (2006)

[2] M. Tomera, "Nonlinear observers design for multivariable ship motion control", Polish Maritime Research. 19, Issue Special, pp. 50-56 (2012)

[3] C. A. Lightcap, S. A. Banks, "An extended Kalman filter for real-time estimation and control of a rigid-link flexible-joint manipulation", IEEE Transactions on Control Systems Technology, no. 1, vol. 8, pp. 91-103 (2010)

[4] B. Sohlberg, "Gray box modeling for model predictive control of a jeating process", Journal of Process Control, no. 3, vol. 13, pp. 225-238 (2003)

[5] H. He, R. Xiong, X. Zhang, F. Sun, \& J. Fan, "State-of-charge estimation of the lithium-ion battery using an adaptive extended Kalman filter based on an improved thevenin model", IEEE Transaction on Vehicular Technology, no. 4, vol. 60, pp. 1461-1469 (2011)

[6] Wang, Weiwen, and Gao Zhiqiang. "A comparison study of advanced state observer design techniques", Proceedings of the IEEE American Control Conference, vol. 6 (2003)

[7] D. Bak, M. Michalik, J. Szafran, "Application of Kalman filter technique to stationary and non-stationary static observer design." IEEE Power Tech Conference Proceedings IEEE Bologna IEEE, vol. 3 (2003)

[8] G. Heredia, A. Ollero, Mechatronics, 2009, "Sensor fault detection in small autonomous helicopter using observer/Kalman filter identification." IEEE International Conference on ICM (2009)

[9] P.S. Nasirabadi, et. al. "Semi-empirical prediction of moisture build-up in an electronic enclosure using analysis of variance (ANOVA)", $18^{\text {th }}$ Electronic Packaging Technology Conference, IEEE (2016)

[10] M. Azzeh, Y. Elseikh, M. Alseid, "An optimized analogy-based project effort estimation", International Journal of Advanced Computer Science and Applications (IJACSA), vol. 5, no. 4, (2014)

[11] H. Nkiama, S.Z.M. Said, M. Saidu, "A subset feature elimination mechanism for intrusion detection system", International Journal of Advanced Computer Science and Applications (IJACSA), vol. 7, no. 4, (2016)

[12] J.N. Rouder et. al., "Model comparison in ANOVA" Psychon Bull Rev, Springer, 23:1779-1786, (2016)

[13] A. R. Khan, M. J. Rabbani, "An ANOVA based predictive parameter estimation", International Conference on Innovations in Electrical Engineering and Computational Technologies (ICIEECT), IEEE, 2017. 